\title{
Gazing at Crystal Balls - Electron Backscatter Diffraction Indexing and Cross Correlation on a Sphere
}

\author{
Ralf Hielscher ${ }^{1}$, Felix Bartel ${ }^{1}$, and T. Ben Britton ${ }^{2}$ \\ 1. Fakultät für Mathematik, Technische Universitat Chemnitz, Chemnitz, Germany. \\ 2. Department of Materials, Imperial College London, London, UK.
}

Electron backscatter diffraction (EBSD) is a common nano- to micro-scale electron microscopy based analysis technique. Rich crystallographic data can be obtained from Kikuchi diffraction based patterns within the scanning electron microscope.

The EBSD pattern is normally captured on a 2D detector inserted within the vacuum chamber and this results in a gnomonic projection of the diffraction pattern. As the EBSD pattern originates from a point source, it can be more interrogated using a spherical frame of reference [1] where the band centers for each diffracting plane form great circles on the sphere.

In this work, we present a new formalism where we approximate the Kikuchi patterns on the sphere. This approximation utilizes fast Fourier algorithms designed for orientation space [2], which are already implemented within MTEX (as they are used for the conventional texture analysis approaches). An example of this approximation is shown in Figure 1.

Once we have the diffraction pattern approximated on the sphere, we can perform a spherical Radon transform to integrate the band profiles (see Figure 2) and generate points of raised intensity which are suitable for indexing using look-up-table based methods such as AstroEBSD [3]. Furthermore, using a variant of this transform we can perform an additional transformation to extract the band profiles of each individual band and present the band profiles for each diffracting plane.

As the diffraction pattern is approximated using a fast Fourier algorithm, which is optimized for $\mathrm{SO}(3)$ space, it is also trivial to perform cross correlation and identify how an experimental pattern (of unknown crystal orientation) projected on the sphere best matches a reference sphere (generated using Dynamical electron simulations).

For both of these approaches, we to be aware that we have incomplete information with respect to our experiment, as it is not possible for the EBSD detector to subtend the entire sphere. Therefore, we apply windowing methods to reduce spectral leakage artifacts.

In this presentation, we will introduce the methods using dynamical simulations to demonstrate accuracy of the method and follow this with a demonstrate of the experimental method using experimental data obtained from pure iron [4].

References:

[1] A. Day, Journal of Microscopy 409 (2008).

[2] R. Hielscher, J. Prestin and A. Vollrath, Mathematical Geosciences 42 (2010), p. 773-794.

[3] T. B. Britton et al., Journal of Applied Crystallography (2018). 
[4] TBB acknowledges funding of his research fellowship from the Royal Academy of Engineering. We thank Alex Foden for useful discussions regarding pattern matching. We thank Jim Hickey for assisting with the example iron data set which was captured in the Harvey Flower EM suite at Imperial College on equipment supported by the Shell AIMS UTC. We thank Aimo Winkelmann for assistance with the spherical re-projection and dynamical pattern generation.
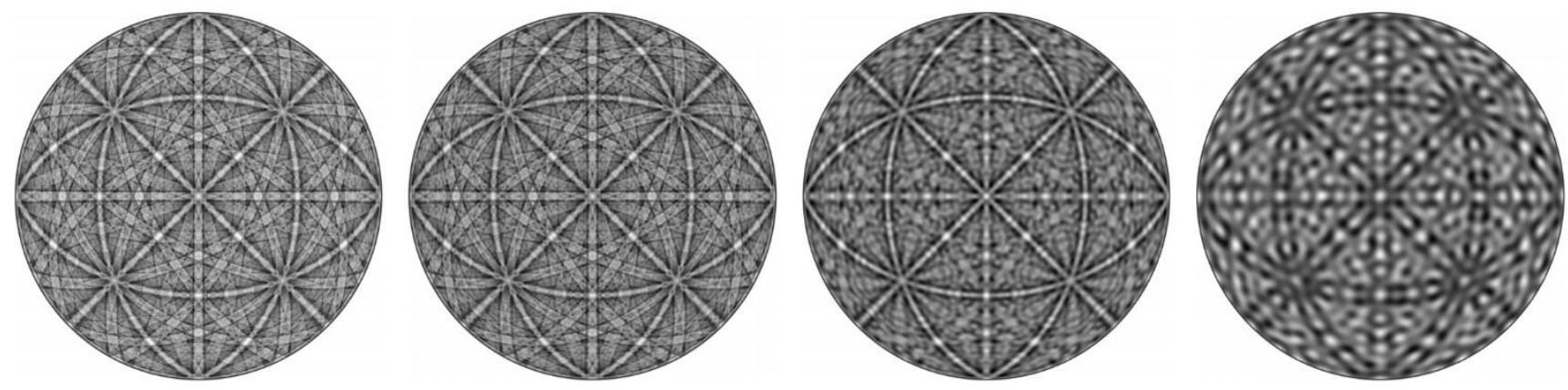

Figure 1. Stereographic projections of the dynamical iron pattern, from left: as simulated; approximated with $\mathrm{N}=256$ harmonics; approximated with $\mathrm{N}=128$ harmonics; approximated with $\mathrm{N}=64$ harmonics.
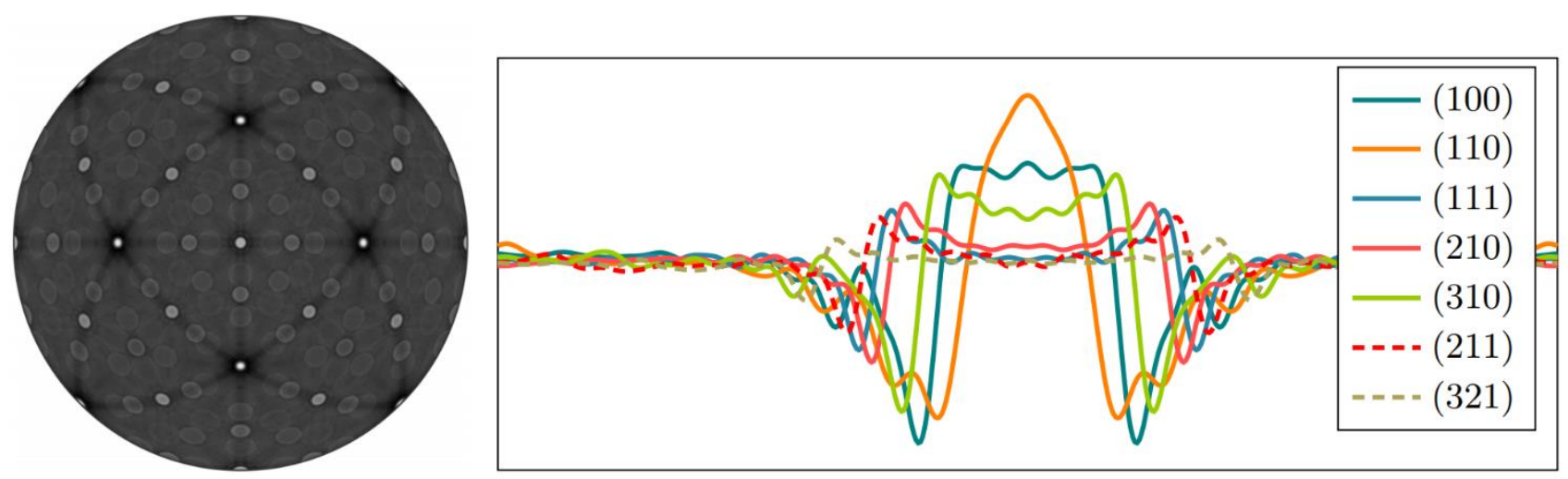

Figure 2. The spherical Radon transform of the iron pattern (left) and the band profiles extracted using integrals around the plane normal within the Radon transform (right). 